\title{
MONOTONICITY OF HYPERBOLIC CURVATURE UNDER UNIVALENT MAPPINGS
}

\author{
Xiangyang Liu and David Minda
}

\begin{abstract}
We investigate the behavior of the hyperbolic (geodesic) curvature of a path on a Riemann surface when the surface increases. Suppose $\mathbf{X}$ is a hyperbolic Riemann surface and $k_{\mathbf{X}}(a, \gamma)$ denotes the hyperbolic curvature at the point $a$ of a smooth path $\gamma$. We determine a necessary and sufficient geometric condition for the existence of a finite constant $K(\mathbf{X})$ such that $k_{\mathbf{X}}(a, \gamma) \leq k_{\mathbf{Y}}(f(a), f \circ \gamma)$ whenever $k_{\mathbf{X}}(a, \gamma) \geq K(\mathbf{X})$ and $f: \mathbf{X} \rightarrow \mathbf{Y}$ is an (injective) conformal embedding of $\mathbf{X}$ into another hyperbolic Riemann surface $\mathbf{Y}$. The constant is independent of $f$ and $\mathbf{Y}$. In particular, this monotonicity property of the hyperbolic curvature holds for any simply connected surface $\mathbf{X}$ with $K(\mathbf{X})=2$; this special case is due to B. Flinn and B. Osgood. They raised the question of considering the problem of monotonicity for hyperbolic curvature for more general surfaces.
\end{abstract}

\section{Introduction}

We begin with a brief review of some topics from hyperbolic geometry. Suppose $\mathbf{X}$ is a hyperbolic Riemann surface; that is, the unit disk $\mathbf{D}$ is the universal covering surface for $\mathbf{X}$. The hyperbolic metric on $\mathbf{D}$, normalized to have Gaussian curvature -1 , is $\lambda_{\mathbf{D}}(z)|d z|=2|d z| /\left(1-|z|^{2}\right)$. If $g: \mathbf{D} \rightarrow \mathbf{X}$ is any analytic universal covering projection, then the hyperbolic metric $\lambda_{\mathbf{X}}(w)|d w|$ on $\mathbf{X}$ is determined from $\lambda_{\mathbf{D}}(z)|d z|=g^{*}\left(\lambda_{X}(w)|d w|\right)$, where $g^{*}\left(\lambda_{\mathbf{X}}(w)|d w|\right)$ is the pull-back of the metric $\lambda_{\mathbf{X}}(w)|d w|$. For a hyperbolic plane region $\mathbf{X}$, we have $\lambda_{\mathbf{X}}(g(z))\left|g^{\prime}(z)\right|=2 /\left(1-|z|^{2}\right)$. The hyperbolic length of a path $\gamma$ on $\mathbf{X}$ is

$$
\operatorname{length}_{\mathbf{x}}(\gamma)=\int_{\gamma} \lambda_{\mathbf{x}}(\mathrm{w})|\mathrm{dw}|
$$

and the hyperbolic distance between the points $a$ and $b$ on $\mathbf{X}$ is

$$
d \mathbf{X}(a, b)=\inf \{\text { length } \mathbf{X}(\gamma): \gamma \text { is a path on } \mathbf{X} \text { joining a and } \mathrm{b}\} .
$$

1980 Mathematics Subject Classification (1985 Revision): 30C35, 30F99,53A30.

Research partially supported by National Science Foundation Grant DMS-9008051. The research of the first author also partially supported by a Student Summer Research Fellowship awarded by the University Research Council of the University of Cincinnati and by a Taft Fellowship awarded by the Taft Foundation. 
The hyperbolic disk with center $a$ and hyperbolic radius $r$ is

$$
D_{\mathbf{X}}(a, r)=\left\{b \in \mathbf{X}: d_{\mathbf{X}}(a, b)<r\right\} .
$$

This paper deals with the behavior of hyperbolic curvature when the surface increases. Let us recall the definition of hyperbolic curvature. First, suppose $\mathbf{X}$ is a hyperbolic region in the complex plane $\mathbf{C}$; that is, $\mathbf{C} \backslash \mathbf{X}$ contains at least two points. If $\gamma: w=w(t), t \in I$ (where $I$ is usually some unspecified interval on the real line) is a $C^{2}$ curve on $\mathbf{X}$, then the hyperbolic curvature of $\gamma$ at a point $w=w\left(t_{0}\right)$ is given by

$$
\kappa_{\mathbf{X}}(w, \gamma)=\frac{1}{\lambda_{\mathbf{X}}(w)}\left\{\kappa_{e}(w, \gamma)+2 \operatorname{Im}\left(\frac{\partial \log \lambda \mathbf{X}(w)}{\partial w} \frac{w^{\prime}\left(t_{0}\right)}{\left|w^{\prime}\left(t_{0}\right)\right|}\right)\right\}
$$

where

$$
\kappa_{e}(w, \gamma)=\frac{1}{\left|w^{\prime}\left(t_{0}\right)\right|} \operatorname{Im}\left(\frac{w^{\prime \prime}\left(t_{0}\right)}{w^{\prime}\left(t_{0}\right)}\right)
$$

denotes the euclidean curvature of $\gamma$ at $w$. Since $\lambda_{\mathbf{D}}(z)=2 /\left(1-|z|^{2}\right)$, we have

$$
\kappa_{\mathbf{D}}(z, \delta)=\frac{1}{2}\left(1-|z|^{2}\right) \kappa_{e}(z, \delta)+\operatorname{Im}\left\{\frac{\overline{z(t)} z^{\prime}(t)}{\left|z^{\prime}(t)\right|}\right\}
$$

for a path $\delta: z=z(t), t \in I$, in $\mathbf{D}$. In particular, $\kappa_{\mathbf{D}}(0, \gamma)=(1 / 2) \kappa_{e}(0, \gamma)$. For a holomorphic universal covering projection $f: \mathbf{D} \rightarrow \mathbf{X}$ and a path $\gamma=f \circ \delta$, we have $\kappa_{\mathbf{D}}(a, \delta)=\kappa_{\mathbf{Y}}(f(a), \gamma)$. This expresses the hyperbolic curvature of a path on $\mathbf{X}$ directly in terms of the hyperbolic curvature of a lifted path on the universal covering surface. In terms of the universal covering projection $f$, we have

$\kappa_{\mathbf{X}}(w, \gamma)=\left(1-|z|^{2}\right)\left|f^{\prime}(z)\right|\left\{\kappa_{e}(w, \gamma)+2 \operatorname{Im}\left(\frac{1}{f^{\prime}(z)}\left[\frac{\bar{z}}{1-|z|^{2}}-\frac{f^{\prime \prime}(z)}{2 f^{\prime}(z)}\right] \frac{w^{\prime}\left(t_{0}\right)}{\left|w^{\prime}\left(t_{0}\right)\right|}\right)\right\}$,

where $w=f(z)$.

The definition of hyperbolic curvature actually makes sense if $\mathbf{X}$ is a hyperbolic Riemann surface, provided one works in terms of local coordinates, because hyperbolic curvature is a conformal invariant. More precisely, hyperbolic curvature is invariant under holomorphic covering projections. That is, $\kappa_{\mathbf{X}}(a, \gamma)=$ $\kappa_{\mathbf{Y}}(f(a), f \circ g)$ if $\mathbf{X}$ and $\mathbf{Y}$ are hyperbolic surfaces and $f: \mathbf{X} \rightarrow \mathbf{Y}$ is an analytic covering projection of $\mathbf{X}$ onto $\mathbf{Y}$.

A number of conformal invariants exhibit monotonic behavior. For instance, the hyperbolic metric itself is monotonic in the sense that $\lambda_{\mathbf{X}}(w)|d w| \geq \lambda_{\mathbf{Y}}(w)|d w|$ on $\mathbf{X}$ if $\mathbf{X} \subset \mathbf{Y}$. Therefore, it is reasonable to inquire about possible monotonicity properties for hyperbolic curvature. The first attempt at establishing a monotonicity property for hyperbolic curvature is due to Haifawa [H]. Unfortunately, there is a gap in his proof. Later, Flinn and Osgood [FO] proved the following monotonicity property of hyperbolic curvature under univalent mappings. 
Flinn-Osgood Monotonicity Theorem. Suppose X, Y are simply connected hyperbolic regions in the complex plane and $f$ is a holomorphic function mapping $\mathbf{X}$ univalently into $\mathbf{Y}$. For any $C^{2}$ curve $\gamma$ on $\mathbf{X}, \kappa_{\mathbf{X}}(a, \gamma) \leq$ $\kappa_{\mathbf{Y}}(f(a), f \circ \gamma)$ if $a$ is any point on $\gamma$ with $\kappa_{\mathbf{X}}(a, \gamma) \geq 2$. The constant 2 is best possible.

Flinn and Osgood also showed that there was no analog of their result for general hyperbolic regions. Explicitly, if $\mathbf{X}=\mathbf{D} \backslash\{0\}, \mathbf{Y}=\mathbf{D}$ and $f$ is the inclusion map of $\mathbf{X}$ into $\mathbf{Y}$, then there is no finite constant $K$ with the property that $\kappa_{\mathbf{X}}(a, \gamma) \leq \kappa_{\mathbf{Y}}(f(a), f \circ \gamma)$ whenever $\kappa_{\mathbf{X}}(a, \gamma) \geq K$. On the other hand, Minda [Mi] showed that the conclusion of the Flinn-Osgood Monotonicity Theorem remains valid for arbitrary hyperbolic Riemann surfaces provided the injective mapping $f$ satisfies an additional condition. The condition is that the group homomorphism $f_{*}$ (induced by $f$ ) from the fundamental group of $\mathbf{X}$ to the fundamental group of $\mathrm{Y}$ should be injective. In particular, the monotonicity property for the hyperbolic metric holds whenever $\mathbf{X}$ is a simply connected hyperbolic Riemann surface and $\mathbf{Y}$ is an arbitrary hyperbolic Riemann surface.

We want to characterize those hyperbolic Riemann surfaces $\mathbf{X}$ for which a version of the Flinn-Osgood Monotonicity Theorem holds. Precisely, we wish to determine those hyperbolic Riemann surfaces $\mathbf{X}$ for which there exists a finite constant $K \geq 0$ ( $K$ depends only on $\mathbf{X}$ ) such that if $f$ is any analytic univalent mapping of $\mathbf{X}$ into another hyperbolic Riemann surface $\mathbf{Y}$, then $\kappa_{\mathbf{X}}(a, \gamma) \leq$ $\kappa_{\mathbf{Y}}(f(a), f \circ \gamma)$ for any $C^{2}$ curve $\gamma$ on $\mathbf{X}$ and all $a$ on $\gamma$ satisfying $\kappa_{\mathbf{X}}(a, \gamma) \geq$ $K$. Precisely, we say that a hyperbolic Riemann surface $\mathbf{X}$ has the hyperbolic curvature monotonicity property, or simply the HCM-property, if there exists a finite constant $K$ such that for all hyperbolic Riemann surfaces $\mathbf{Y}$, all $C^{2}$ curves $\gamma$ on $\mathbf{X}$ and all $a$ on $\gamma, \kappa_{\mathbf{X}}(a, \gamma) \geq K$ implies $\kappa_{\mathbf{X}}(a, \gamma) \leq \kappa_{\mathbf{Y}}(f(a), f \circ \gamma)$ for any univalent analytic function $f: \mathbf{X} \rightarrow \mathbf{Y}$. Let $K(\mathbf{X})$ be the infimum (in fact, the minimum) of all such $K$. Define $K(\mathbf{X})=\infty$ if $\mathbf{X}$ does not have the HCMproperty. In other words, $\mathbf{X}$ has the HCM-property if and only if $K(\mathbf{X})<\infty$.

Thus, our goal is to characterize hyperbolic Riemann surfaces with the HCMproperty. We say that $\mathrm{X}$ can be embedded in $\mathrm{Y}$ if there exists a univalent mapping from $\mathbf{X}$ to $\mathbf{Y}$; frequently we regard $\mathbf{X}$ as as a subset of $\mathbf{Y}$ when $\mathbf{X}$ is embedded in $\mathbf{Y}$. One difficulty in attempting to characterize surfaces with the HCM-property is that certain surfaces possess this property for a trivial reason. A hyperbolic Riemann surface is called maximal hyperbolic if it cannot be properly embedded into another hyperbolic Riemann surface. Clearly, if $\mathbf{X}$ is maximal hyperbolic, then $\mathbf{X}$ has the HCM-property and $K(\mathbf{X})=0$. In fact, hyperbolic curvature on a maximal hyperbolic Riemann surface $\mathbf{X}$ is unchanged under a univalent mapping $f$ of $\mathbf{X}$ into another hyperbolic surface $\mathbf{Y}$ because any such mapping is actually a conformal mapping of $\mathbf{X}$ onto $\mathbf{Y}$. In order to discuss the possible monotonicity of the hyperbolic curvature, it is reasonable to determine which hyperbolic Riemann surfaces are maximal. In Section 3 we present a complete list 
of maximal hyperbolic surfaces with finite genus.

The characterization of hyperbolic surfaces with the HCM-property involves the concept of a uniformly perfect Riemann surface. Recall that a hyperbolic plane region $\mathbf{X}$ is called uniformly perfect if there exists $c>0$ such that $\lambda \mathbf{X} \geq c / \delta_{\mathbf{X}}$, where $\delta_{\mathbf{X}}(w)$ denotes the euclidean distance between $w$ and the boundary of $\mathbf{X}$. We introduce a natural extension of this concept for hyperbolic Riemann surfaces and show that it is a sufficient condition for a Riemann surface to have the HCMproperty. In Section 4 we show that a hyperbolic Riemann surface with finite genus has the HCM-property if and only if the surface is either maximal hyperbolic or uniformly perfect. Also, in Section 4 a necessary and sufficient geometric condition for a surface of arbitrary genus to have the HCM-property is given.

We want to thank the referee for carefully reading the manuscript and making several useful suggestions for improving the exposition.

\section{Uniformly perfect Riemann surfaces}

From now on $\mathbf{X}$ will always denote a hyperbolic Riemann surface, and we assume all mappings between Riemann surfaces are analytic.

We want to extend the concept of uniform perfectness to a hyperbolic Riemann surface. Suppose $f$ is an analytic mapping from $\mathbf{X}$ to another Riemann surface. For $z \in \mathbf{X}$, let $s(z, f)$ be the supremum of all $s$ such that $f$ is univalent in the hyperbolic disk $D_{\mathbf{X}}(z, s)$. Set $s(f)=\inf \{s(z, f): z \in \mathbf{X}\}$. Then $f$ is called uniformly locally univalent (in the hyperbolic sense) if $s(f)>0$. The quantity $s(f)$ is called the hyperbolic radius of uniform univalence for $f$.

For a hyperbolic plane region, we recall that the notion of uniform perfectness is equivalent to uniform local univalence of any universal covering projection. Let $\mathbf{X}$ be a hyperbolic plane region and $g: \mathbf{D} \rightarrow \mathbf{X}$ be a covering projection. It is known (see $\left[\mathrm{Po}_{2}\right]$ ) that $\mathbf{X}$ is uniformly perfect if and only if $g$ is uniformly locally univalent.

There is a hyperbolic geometric interpretation for the uniform local univalence of a universal covering projection. For $a \in \mathbf{X}$, let $r_{\mathbf{X}}(a)$ be the supremum of all $r$ such that the hyperbolic disk $D_{\mathbf{X}}(a, r)$ is simply connected. Suppose that $g: \mathbf{D} \rightarrow \mathbf{X}$ is a covering projection. It is easy to see that if $z \in \mathbf{D}$ satisfies $g(z)=a$, then $r_{\mathbf{X}}(a)=s(z, g)$. If we introduce the quantity $r(\mathbf{X})=\inf \left\{r_{\mathbf{X}}(a): a \in \mathbf{X}\right\}$, then $r(\mathbf{X})=s(g)$. The quantity $r(\mathbf{X})$ is called the hyperbolic radius of injectivity. Consequently, the following definition is a reasonable extension of the concept of uniform perfectness to a hyperbolic Riemann surface.

Definition. A hyperbolic Riemann surface $\mathbf{X}$ is called uniformly perfect if $r(\mathbf{X})>0$.

Clearly, the notion of uniform perfectness is conformally invariant. Note that all simply connected hyperbolic Riemann surfaces are uniformly perfect according to this definition (if $\mathbf{X}$ is simply connected, then $r(\mathbf{X})=\infty$ ). We want to mention 
the following known fact without a proof [BP]: A plane region with an isolated boundary point is not uniformly perfect.

Example. Let $\varrho \in(0,1)$ and $A(\varrho)$ be the annulus $\{z: \varrho<|z|<1\}$. Any hyperbolic doubly-connected plane region is conformally equivalent to $A(\varrho)$ for some $\varrho$. Direct calculation shows that $r(A(\varrho))=\pi^{2} /(\log \varrho)=\pi /(2 \bmod (A(\varrho)))$, where $\bmod (A(\varrho))$ is the conformal modulus of $A(\varrho)$. Therefore, a doubly connected hyperbolic plane region is uniformly perfect if and only if its conformal modulus is finite.

For holomorphic functions mapping $\mathbf{D}$ into either itself or $\mathbf{C}$, the idea of uniform local univalence is closely related to two "norms". We know that a holomorphic function $F$ is locally univalent if its derivative has no zero. If $F$ is locally univalent in $\mathbf{D}$, let

$$
L(F, z)=\left|\frac{1}{2}\left(1-|z|^{2}\right) F^{\prime \prime}(z) / F^{\prime}(z)-\bar{z}\right| .
$$

It is easy to verify that $L(F \circ T, z)=L(F, T(z))$ for all conformal automorphisms $T$ of $\mathbf{D}$. Define the linear invariant norm ( $L$-norm) of $F$ by

$$
\|F\|_{L}=\sup \{L(F, z): z \in \mathbf{D}\}
$$

The above observation shows that $\|F\|_{L}=\|F \circ T\|_{L}$ if $T$ is a conformal automorphism of $\mathbf{D}$. By calculation, $\|F\|_{L}=1$ if $F$ itself is a conformal automorphism of $\mathbf{D}$. In fact, $\|F\|_{L}=1$ if and only if $F$ is a convex univalent function [ $\left[\mathrm{Po}_{1}\right]$.

If $F$ is a locally univalent mapping of $\mathbf{D}$ into $\mathbf{D}$ and $F$ is not a conformal automorphism of $\mathbf{D}$, then let $H(F, z)=H_{2}(F, z) / H_{1}(F, z)$, where

$$
\begin{aligned}
& H_{1}(F, z)=1-\frac{\left|F^{\prime}(z)\right|\left(1-|z|^{2}\right)}{1-|F(z)|^{2}}, \\
& H_{2}(F, z)=\left|\frac{1}{2}\left(1-|z|^{2}\right) \frac{F^{\prime \prime}(z)}{F^{\prime}(z)}-\bar{z}+\frac{\overline{F(z)} F^{\prime}(z)\left(1-|z|^{2}\right)}{1-|F(z)|^{2}}\right| .
\end{aligned}
$$

Note that $H_{1}(F, z)>0$ is the invariant form of Schwarz' Lemma. The hyperbolic linear invariant norm ( $H$-norm) of $F$ is given by

$$
\|F\|_{H}=\sup \{H(F, z): z \in \mathbf{D}\} .
$$

It is not difficult to verify that $H(S \circ(F \circ T), z)=H(F, T(z))$ if $S$ and $T$ are conformal automorphisms of $\mathbf{D}$, so $\|F\|_{H}=\|S \circ(F \circ T)\|_{H}$.

Note that the $L$-norm and the $H$-norm are not really "norms" in the linear algebra sense. 
Since $H_{1}(F, z)=0$ if $F$ is a conformal automorphism of $\mathbf{D}$, we cannot define $\|F\|_{H}$ as above. For each $t \in(0,1)$, let $F_{t}(z)=F(t z)$. Then $F_{t}$ is not a conformal automorphism of $\mathbf{D}$, so $\left\|F_{t}\right\|_{H}$ is well-defined. We define $\|F\|_{H}$ to be the limit of $\left\|F_{t}\right\|_{H}$ as $t$ increases to 1 . In order to justify this definition, we have to show that the above limit exists, and equals $\|F\|_{H}$ when $F$ is not a conformal automorphism of $\mathbf{D}$. Fortunately, these facts are easy to verify; we omit the details because we do not really need to use the concept of $H$-norm for conformal automorphisms of $\mathbf{D}$ in this paper. Direct calculation shows that any conformal automorphism of $\mathbf{D}$ has $H$-norm 1. See [L] for details.

Lemma 1. Suppose that $F$ is a locally univalent function of $\mathbf{D}$ into $\mathbf{D}$. Then the two norms $\|F\|_{H}$ and $\|F\|_{L}$ are equivalent. In fact,

$$
1 \leq\|F\|_{L} \leq\|F\|_{H} \leq 2\|F\|_{L}+1 \leq 3\|F\|_{L} .
$$

Proof. If $F$ is a conformal automorphism of $\mathbf{D}$, then the results are trivial because $\|F\|_{L}=\|F\|_{H}=1$. So we need only to consider the case when $F$ is not a conformal automorphism of $\mathrm{D}$.

The inequality $1 \leq\|F\|_{L}$ is due to Pommerenke $\left[\mathrm{Po}_{1}\right]$, while $\|F\|_{L} \leq\|F\|_{H}$ is due to $\mathrm{Ma}$ and Minda $\left(\left[\mathrm{MM}_{1}\right],\left[\mathrm{MM}_{2}\right]\right)$. We need only to show that $\|F\|_{H} \leq$ $2\|F\|_{L}+1$.

For each $a \in \mathbf{D}$, let $G(z)=(S \circ(F \circ T))(z)$, where $T(z)=(z+a) /(1+\bar{a} z)$ and $S(z)=(F(z)-F(a)) /(1-\overline{F(a)} F(z))$. Then $G$ maps $\mathbf{D}$ into $\mathbf{D}, G(0)=0$ and $\left|G^{\prime}(0)\right|<1$. By a straightforward application of the Schwarz-Pick Lemma, we obtain $\left|G^{\prime \prime}(0) \leq 2\right|\left(1-\left|G^{\prime}(0)\right|^{2}\right)$. From direct calculation, we have

$$
\begin{aligned}
& H_{1}(F, a)=1-\left|G^{\prime}(0)\right|, \\
& H_{2}(F, a)=\left|G^{\prime \prime}(0)\right| /\left(2\left|G^{\prime}(0)\right|\right),
\end{aligned}
$$

and

$$
H(F, a)=\left|G^{\prime \prime}(0)\right| /\left(2\left|G^{\prime}(0)\right|\left(1-\left|G^{\prime}(0)\right|\right)\right) .
$$

If $\left|G^{\prime}(0)\right| \geq 1 / 2$, then $\left|G^{\prime \prime}(0)\right| \leq 2\left(1-\left|G^{\prime}(0)\right|^{2}\right)$ implies that

$$
\begin{aligned}
H(F, a) & =\left|G^{\prime \prime}(0)\right| /\left(2\left|G^{\prime}(0)\right|\left(1-\left|G^{\prime}(0)\right|\right)\right) \\
& \leq\left(1+\left|G^{\prime}(0)\right|\right) /\left|G^{\prime}(0)\right| \leq 3 \leq 2\|F\|_{L}+1 .
\end{aligned}
$$

On the other hand, if $\left|G^{\prime}(0)\right|<1 / 2$, then

$$
\begin{aligned}
H(F, a) & =H_{2}(F, a) /\left(1-\left|G^{\prime}(0)\right|\right) \leq\left(L(F, a)+\left|G^{\prime}(0)\right|\right) /\left(1-\left|G^{\prime}(0)\right|\right) \\
& \leq 2\left(L(F, a)+\left|G^{\prime}(0)\right|\right) \leq 2 L(F, a)+1 \leq 2\|F\|_{L}+1 .
\end{aligned}
$$

Therefore, $\|F\|_{H} \leq 2\|F\|_{L}+1$. 
Lemma 2. Suppose that $F$ is a locally univalent function of $\mathbf{D}$ into $\mathbf{D}$. Then

$$
\frac{1}{\tanh (s(F) / 2)} \leq\|F\|_{H} \leq \frac{2}{\tanh (s(F) / 2)} .
$$

Proof. We first establish the upper bound. We may assume that $s(F)>0$. If $G$ is defined as in the proof of Lemma 1 , then $G$ is still univalent in each hyperbolic disk of radius $r=s(F)$. In particular, $G$ is univalent in the euclidean disk centered at 0 with radius $R=\tanh (r / 2)$. Then the function $g(z)=G(R z) / R$ is univalent in $\mathbf{D}$ and maps $\mathbf{D}$ into $\mathbf{D}$. A result of Pick $[\mathrm{P}]$ gives

$$
\left|g^{\prime \prime}(0)\right| \leq 4\left|g^{\prime}(0)\right|\left(1-\left|g^{\prime}(0)\right|\right) \text {. }
$$

Therefore,

$$
\begin{aligned}
H(F, a) & =\left|G^{\prime \prime}(0)\right| /\left(2\left|G^{\prime}(0)\right|\left(1-\left|G^{\prime}(0)\right|\right)\right) \\
& =\left|g^{\prime \prime}(0)\right| /\left(2 R\left|g^{\prime}(0)\right|\left(1-\left|g^{\prime}(0)\right|\right)\right) \leq 2 / R .
\end{aligned}
$$

So $\|F\|_{H} \leq 2 / \tanh (r / 2)$.

Next we derive the lower bound. For any $a \in \mathbf{D}$, let $G(z)=(F \circ T)(z)$, where $T(z)=(z+a) /(1+\bar{a} z)$. Then

$$
L(G, z) \leq\|G\|_{L}=\|F\|_{L} \leq\|F\|_{H}=m
$$

implies that

$$
\operatorname{Re}\left\{1+z G^{\prime \prime}(z) / G^{\prime}(z)\right\} \geq 0, \quad|z|<m-\sqrt{m^{2}-1}
$$

Thus, $G$ is convex univalent in the euclidean disk centered at 0 with radius $m-$ $\sqrt{m^{2}-1}$. If we can show that $G$ is univalent in the euclidean disk centered at 0 with radius $1 / m$, then $F$ will be univalent in the hyperbolic disk centered at $a$ with radius $r=2 \operatorname{artanh}(1 / m)$. That is, $s(F) \geq r$. So it is enough to prove that for each fixed $z \in \mathbf{D}$ with $|z|<1 / m$,

$$
\operatorname{Re}\left\{z G^{\prime}(z) /[G(z)-G(0)]\right\} \geq 0 .
$$

Select $w \in \mathbf{D}$ such that $z=2 w /\left(1+|w|^{2}\right)$, then

$$
|w|=1 /|z|-\sqrt{1 /|z|^{2}-1}<m-\sqrt{m^{2}-1} .
$$

By the same argument as above, we know that the function

$$
E(u)=G((u+w) /(1+\bar{w} u))
$$


is convex univalent in the disk $\left\{u:|u|<m-\sqrt{m^{2}-1}\right\}$. Therefore, the image of the disk $\left\{u:|u|<m-\sqrt{m^{2}-1}\right\}$ under the mapping $E$ is starlike with respect to $E(-w)$. In particular, we have

$$
\operatorname{Re}\left\{z G^{\prime}(z) /[G(z)-G(0)]\right\}=\frac{2\left(1+|w|^{2}\right)}{1-|w|^{2}} \operatorname{Re}\left\{w E^{\prime}(w) /[E(w)-E(-w)]\right\} \geq 0
$$

Lemma 3. Suppose $\mathbf{X}$ and $\mathbf{Y}$ are hyperbolic Riemann surfaces, $f: \mathbf{X} \rightarrow \mathbf{Y}$ is analytic and locally univalent but not a covering of $\mathbf{X}$ onto $\mathbf{Y}$ and $a \in \mathbf{X}$. Assume $g: \mathbf{D} \rightarrow \mathbf{X}$ and $h: \mathbf{D} \rightarrow \mathbf{Y}$ are covering projections satisfying $g(0)=a$ and $h(0)=f(a)$ and $F: \mathbf{D} \rightarrow \mathbf{D}$ is the unique holomorphic function satisfying $F(0)=0$ and $f \circ g=h \circ F$.

(i) If $\gamma$ is a $C^{2}$ curve on $\mathbf{X}$ through $a$, then $\kappa_{\mathbf{X}}(a, \gamma) \leq \kappa_{\mathbf{Y}}(f(a), f \circ \gamma)$ provided that $\kappa_{\mathbf{X}}(a, \gamma) \geq H(F, 0)$.

(ii) Suppose there exists a positive number $K$ with the property that $\kappa_{\mathbf{X}}(a, \gamma) \leq$ $\kappa_{\mathbf{Y}}(f(a), f \circ \gamma)$ whenever $\kappa_{\mathbf{X}}(a, \gamma) \geq K$. Then $K \geq H(F, 0)$.

Proof. Since $f$ is not a covering of $\mathbf{X}$ onto $\mathbf{Y}$, the function $F$ is not a conformal automorphism of D. In particular, $\left|F^{\prime}(0)\right|<1$.

(i) Let $\delta: z=z(t)$ be the lift of $\gamma$ via $g$ passing through 0 with $z(0)=0$. By definition, we have

$$
\kappa_{\mathbf{X}}(a, \gamma)=\kappa_{\mathbf{D}}(0, \delta)=(1 / 2) \kappa_{e}(0, \delta)
$$

and

$$
\kappa_{\mathbf{Y}}(f(a), f \circ \gamma)=\kappa_{\mathbf{D}}(0, F \circ \delta)=(1 / 2) \kappa_{e}(0, F \circ \delta) .
$$

By calculation we obtain

$$
\left|F^{\prime}(0)\right| \kappa_{e}(0, F \circ \delta)=\kappa_{e}(0, \delta)+\operatorname{Im}\left[\frac{F^{\prime \prime}(0)}{F^{\prime}(0)} \frac{z^{\prime}(0)}{\left|z^{\prime}(0)\right|}\right] \geq \kappa_{e}(0, \delta)-\left|\frac{F^{\prime \prime}(0)}{F^{\prime}(0)}\right| .
$$

Note that equality can hold in inequality (1) for an appropriate choice of $\gamma$. Since $\left|F^{\prime}(0)\right|<1$, then the above inequality is equivalent to

$$
\left|F^{\prime}(0)\right|\left[\kappa_{\mathbf{Y}}(f(a), f \circ \gamma)-H(F, 0)\right] \geq \kappa_{\mathbf{X}}(a, \gamma)-H(F, 0) .
$$

Inequality (2) implies that $\kappa_{\mathbf{X}}(a, \gamma) \leq \kappa_{\mathbf{Y}}(f(a), f \circ \gamma)$ whenever $\kappa_{\mathbf{X}}(a, \gamma) \geq$ $H(F, 0)$.

(ii) For any value of $\kappa$ we can select a path $\gamma$ through $a$ with $\kappa_{\mathbf{X}}(a, \gamma)=\kappa$ such that equality holds in inequality (1). Then

$$
\left|F^{\prime}(0)\right|\left[\kappa_{\mathbf{Y}}(f(a), f \circ g)-H(F, 0)\right]=\kappa_{\mathbf{X}}(a, \gamma)-H(F, 0) .
$$

If $\kappa_{\mathbf{X}}(a, \gamma)=\kappa<H(F, 0)$, then the preceding inequality in conjunction with $\left|F^{\prime}(0)\right|<1$ gives $\kappa_{\mathbf{X}}(a, \gamma)>\kappa_{\mathbf{Y}}(f(a), f \circ \gamma)$. Consequently, $K \geq H(F, 0)$. 
Theorem 1. Suppose that $\mathbf{X}$ is uniformly perfect. Then $\mathbf{X}$ has the HCMproperty and

$$
K(\mathbf{X}) \leq 2 / \tanh (r(\mathbf{X}) / 2) .
$$

Proof. Let $f$ be a univalent analytic mapping of $\mathbf{X}$ into another hyperbolic Riemann surface $\mathbf{Y}$ and let $\gamma$ be a $C^{2}$ curve on $\mathbf{X}$. There is no harm in assuming that $f$ is not a conformal mapping of $\mathbf{X}$ onto $\mathbf{Y}$. For any $a$ on $\gamma$, let $g: \mathbf{D} \rightarrow \mathbf{X}$ and $h: \mathbf{D} \rightarrow \mathbf{Y}$ be covering projections satisfying $g(0)=a$ and $h(0)=f(a)$. Then there is an $F: \mathbf{D} \rightarrow \mathbf{D}$ satisfying $F(0)=0$ and $f \circ g=h \circ F$. Since $f$ is univalent and $\mathbf{X}$ is uniformly perfect, $r(\mathbf{X})>0$, and so $g$ (and hence $f \circ g)$ is univalent in each hyperbolic disk of radius $r(\mathbf{X})$. This implies that $F$ is univalent in each hyperbolic disk of radius $r(\mathbf{X})$. From Lemma 2, we obtain $\|F\|_{H} \leq 2 / \tanh (r(\mathbf{X}) / 2)$. By Lemma 3(i), $\kappa_{\mathbf{X}}(a, \gamma) \leq \kappa_{\mathbf{Y}}(f(a), f \circ \gamma)$ provided that $\kappa_{\mathbf{X}}(a, \gamma) \geq H(F, 0)$. In particular, the hyperbolic curvature will increase if $\kappa_{\mathbf{X}}(a, \gamma) \geq\|F\|_{H}$. Because $\|F\|_{H} \leq 2 / \tanh (r(\mathbf{X}) / 2)$, we obtain $K(\mathbf{X}) \leq 2 / \tanh (r(\mathbf{X}) / 2)$. This completes the proof.

Remark. In the case $\mathbf{X}$ is simply connected, Theorem 1 gives $K(\mathbf{X}) \leq 2$ since $r(\mathbf{X})=\infty$; this is the Flinn-Osgood Monotonicity Theorem.

Theorem 1 asserts that uniform perfectness is a sufficient condition for the HCM-property. The next result shows that this condition is also necessary when $\mathbf{X}$ can be embedded in a uniformly perfect hyperbolic Riemann surface.

Theorem 2. Suppose that $f$ maps $\mathbf{X}$ univalently into $\mathbf{Y}$ and $\mathbf{Y}$ is uniformly perfect. If there exists a constant $K>0$ ( $K$ may depend on both $\mathbf{X}$ and $f$ ) such that

$$
\kappa_{\mathbf{X}}(a, \gamma) \leq \kappa_{\mathbf{Y}}(f(a), f \circ \gamma)
$$

for all $C^{2}$ curves $\gamma$ on $\mathbf{X}$ and any $a$ on $\gamma$ satisfying $\kappa_{\mathbf{X}}(a, \gamma) \geq K$, then $\mathbf{X}$ is uniformly perfect.

Proof. Let $g: \mathbf{D} \rightarrow \mathbf{X}$ and $h: \mathbf{D} \rightarrow \mathbf{Y}$ be covering projections. Then there is a function $F: \mathbf{D} \rightarrow \mathbf{D}$ such that $f \circ g=h \circ F$. First, we want to show that $F$ is uniformly locally univalent. If $f$ is a covering of $\mathbf{X}$ onto $\mathbf{Y}$, then $F$ is a conformal automorphism of $\mathbf{D}$ and so $F$ is actually univalent. Now, suppose $f$ is not a covering.

For each $w \in \mathbf{D}$, set $a=g(w)$ and let $T$ be a conformal automorphism of D mapping $w$ to 0 and $S$ be a conformal automorphism of $\mathbf{D}$ mapping $F(w)$ to 0 . Then the function $F_{a}=S \circ\left(F \circ T^{-1}\right)$ is a lifting of $f$ (via the covering projections $g \circ T^{-1}$ and $h \circ S^{-1}$ ), and $F_{a}(0)=0$. From Lemma 3(ii) we obtain

$$
K \geq H\left(F_{a}, 0\right)=H(F, w) .
$$

This holds for all $w$ in $\mathbf{D}$, so $\|F\|_{H} \leq K$. By Lemma $2, F$ is uniformly locally univalent and $s(F) \geq 2 \operatorname{artanh}(1 / K)$. 
The Schwarz-Pick Lemma shows that $F$ maps a hyperbolic disk centered at $z \in \mathbf{D}$ into a hyperbolic disk centered at $F(z)$ with the same hyperbolic radius. Since $h$ is also uniformly locally univalent, $h \circ F$ is uniformly locally univalent. The relation $f \circ g=h \circ F$ and the univalence of $f$ imply that $g$ is uniformly locally univalent. Therefore, $r(\mathbf{X})=s(g)>0$, and so $\mathbf{X}$ is uniformly perfect.

We give another characterization of uniformly perfect Riemann surfaces which is needed later. Let $\Gamma(\mathbf{X})$ be the family of all homotopically nontrivial closed $C^{2}$ curves on $\mathbf{X}$. Define

$$
\mathcal{M}(\mathbf{X})=\inf \left\{\text { length }_{\mathbf{X}}(\gamma): \gamma \in \Gamma(\mathbf{X})\right\}
$$

if $\Gamma(\mathbf{X})$ is nonempty, and

$$
\mathcal{M}(\mathrm{X})=\infty
$$

if $\Gamma(\mathbf{X})$ is empty.

Theorem 3. $\mathcal{M}(\mathbf{X})=2 r(\mathbf{X})$. Therefore, $\mathbf{X}$ is uniformly perfect if and only if

$$
\mathcal{M}(\mathrm{X})>0 .
$$

Proof. Let $c=\mathcal{M}(\mathbf{X})$ and $g: \mathbf{D} \rightarrow \mathbf{X}$ be a covering projection. Note that $s(g)=r(\mathbf{X})$, so it suffices to show that $s(g)=c / 2$.

If $s(g)<c / 2$, then there are two distinct points $z$ and $z^{\prime}$ in $\mathbf{D}$ with $d_{\mathbf{D}}\left(z, z^{\prime}\right)<c$ and $g(z)=g\left(z^{\prime}\right)$. Let $\delta$ be the geodesic joining $z$ and $z^{\prime}$. Then the closed path $\gamma=g \circ \delta$ is homotopically nontrivial since $\delta$ is not closed and so belongs to $\Gamma(\mathbf{X})$. But,

$$
\text { length }_{\mathbf{X}}(\gamma)=\text { length }_{\mathbf{D}}(\delta)<\mathrm{c},
$$

a contradiction.

If $s(g)>c / 2$, then $\Gamma(\mathbf{X})$ is nonempty and there exists a $\gamma \in \Gamma(\mathbf{X})$ such that length $\mathbf{x}(\gamma)<2 \mathrm{~s}(\mathrm{~g})$. Let $\delta$ be a lifting of $\gamma$ via $g$ with initial point $z$ and end point $z^{\prime}$. Then $z \neq z^{\prime}$ since $\gamma$ is homotopically nontrivial on $\mathbf{X}$. The fact that $\gamma$ is closed implies $g(z)=g\left(z^{\prime}\right)$. So

$$
2 s(g) \leq \text { length }_{\mathrm{D}}(\delta)=\text { length }_{\mathrm{X}}(\gamma)<2 \mathrm{~s}(\mathrm{~g}) .
$$

This is impossible. 


\section{Maximal hyperbolic Riemann surfaces}

We begin by identifying some maximal hyperbolic surfaces. Note that a maximal hyperbolic surface need not be uniformly perfect since the first two types of maximal hyperbolic surfaces in Theorem 4 are not uniformly perfect. It seems that the results of this section might be known, especially to experts, but we were unable to locate the results in the literature and have included complete proofs for the benefit of the reader.

Theorem 4. If $\mathbf{X}$ is a thrice punctured Riemann sphere, a once punctured torus, or a compact Riemann surface of finite genus $\mathbf{g} \geq 2$, then $\mathbf{X}$ is maximal hyperbolic.

Proof. Let $\mathbf{Y}$ be a hyperbolic Riemann surface and $f$ be a univalent analytic mapping from $\mathbf{X}$ to $\mathbf{Y}$. We only need to show that $f$ is surjective. We consider separately the three different types of surfaces.

First, suppose $\mathbf{X}$ is compact with genus $\mathbf{g} \geq 2$. Then the image of $\mathbf{X}$ under $f$ is both open and closed in $\mathbf{Y}$, so $f$ must be surjective.

Next, suppose $\mathbf{X}$ is a once punctured torus. By Ohtsuka's generalization of Picard's Theorem ([O], [MRR]), $f$ extends to a holomorphic mapping $f_{1}$ defined on the torus, say $\mathbf{X}_{1}=\mathbf{X} \cup\{p\}$, and $f_{1}$ maps $\mathbf{X}_{1}$ into $\mathbf{Y}_{1}$, where $\mathbf{Y}_{1}$ is either $\mathbf{Y}$ or $\mathbf{Y}$ with one isolated boundary point added. By using the fact that $\mathbf{X}_{1}$ is compact, we obtain $f_{1}\left(\mathbf{X}_{1}\right)=\mathbf{Y}_{1}$. But a nonconstant analytic mapping from one compact Riemann surface onto another must be $n$-to- 1 for some integer $n \geq 1$. Since $f_{1}$ is univalent on $\mathbf{X}=\mathbf{X}_{1} \backslash\{p\}$, we must have $n=1$. Thus, $f(\mathbf{X})=\mathbf{Y}_{1} \backslash\left\{f_{1}(p)\right\}=\mathbf{Y}$ and so $f$ is surjective.

Finally, consider the case in which $\mathbf{X}=\mathbf{P} \backslash\{a, b, c\}$, where $\mathbf{P}$ denotes the Riemann sphere. Now, Ohtsuka's generalization of Picard's Theorem implies that $f$ extends to an analytic mapping $f_{1}: \mathbf{P} \rightarrow \mathbf{Y}_{1}$, where $\mathbf{Y}_{1}$ is either $\mathbf{Y}$ or $\mathbf{Y}$ with one, two or three isolated boundary points added. By using a similar argument as in the preceding case, we may conclude that $f$ is surjective.

Lemma 4. Every hyperbolic Riemann surface can be embedded in a maximal hyperbolic Riemann surface.

Proof. Let $\mathbf{X}$ be a hyperbolic Riemann surface. We consider the cases of finite and infinite genus separately.

If $\mathbf{X}$ has finite genus $\mathbf{g}$, then by a result of Maskit [M], $\mathbf{X}$ can be embedded in a compact Riemann surface $\mathbf{Z}$ of the same genus. We may regard $\mathbf{X}$ as a subset of $\mathbf{Z}$. We treat the cases $\mathbf{g}=0, \mathbf{g}=1$ and $\mathbf{g} \geq 2$ separately. First, suppose $\mathbf{g}=0$. Note that $\mathbf{g}=0$ implies that $\mathbf{Z}$ is conformally equivalent to the Riemann sphere; hence $\mathbf{Z} \backslash \mathbf{X}$ must contain three or more points to make $\mathbf{X}$ hyperbolic. Let $\{a, b, c\}$ be a set of three distinct points in $\mathbf{Z} \backslash \mathbf{X}$. Then $\mathbf{Y}=\mathbf{Z} \backslash\{a, b, c\}$ is maximal hyperbolic and $\mathbf{X}$ can be embedded into $\mathbf{Y}$. For the case $\mathbf{g}=1, \mathbf{Z}$ is conformally equivalent to a torus, so $\mathbf{Z} \backslash \mathbf{X}$ must be nonempty since $\mathbf{Z}$ is not 
hyperbolic. Let $a \in \mathbf{Z} \backslash \mathbf{X}$, then $\mathbf{Y}=\mathbf{Z} \backslash\{a\}$ is maximal hyperbolic and $\mathbf{X}$ can be embedded into $\mathbf{Y}$. If $\mathbf{g} \geq 2$, then $\mathbf{Z}$ itself is maximal hyperbolic and there is nothing more to prove.

If $\mathbf{X}$ has infinite genus, then $\mathbf{X}$ is not compact. A result of Bochner [B] says that $\mathbf{X}$ can be embedded into a Riemann surface $\mathbf{Y}$ such that $\mathbf{Y}$ cannot be embedded properly into any other Riemann surface. The surface $\mathbf{Y}$ must be hyperbolic since it cannot have finite genus, so $\mathbf{Y}$ is maximal hyperbolic.

The following theorem determines all maximal hyperbolic Riemann surfaces with finite genus.

Theorem 5. If $\mathbf{X}$ is a maximal hyperbolic Riemann surface with finite genus $\mathbf{g}$, then $\mathbf{X}$ is conformally equivalent to either a thrice-punctured Riemann sphere, a once-punctured torus, or a compact Riemann surface with $\mathbf{g} \geq 2$.

Proof. Recall that $\mathbf{X}$ can be embedded into a compact Riemann surface $\mathbf{Z}$ of genus $\mathbf{g}[\mathrm{M}]$. We may consider $\mathbf{X}$ as a subset of $\mathbf{Z}$. If $\mathbf{g}=0$, then $\mathbf{Z}$ is conformally equivalent to the Riemann sphere, and $\mathbf{Z} \backslash \mathbf{X}$ consists of exactly three points since $\mathbf{X}$ is maximal hyperbolic. For if $\mathbf{Z} \backslash \mathbf{X}$ had more than three points, let $a \in \mathbf{Z}$ be a boundary point of $\mathbf{X}$. Then for a sufficiently small $\varepsilon>0$, $\mathbf{Y}=\mathbf{X} \cup\left\{z \in \mathbf{Z}: d_{\mathbf{Z}}(z, a)<\varepsilon\right\}$ would be a hyperbolic Riemann surface and $\mathbf{X}$ could be properly embedded into $\mathbf{Y}$. This is impossible. If $\mathbf{g}=1$, then $\mathbf{Z}$ is conformally equivalent to a torus. By the same argument as in the case $\mathbf{g}=0$, we deduce that $\mathbf{Z} \backslash \mathbf{X}$ consists of exactly one point. If $\mathbf{g} \geq 2$, then $\mathbf{Z}$ itself is a hyperbolic Riemann surface, so $\mathbf{X}$ equals $\mathbf{Z}$ since $\mathbf{X}$ is maximal.

\section{Characterization of surfaces with the HCM-property}

We give two characterizations of hyperbolic surfaces with the HCM-property. The first applies to surfaces of finite genus while the second is for arbitrary hyperbolic surfaces.

Theorem 6. Suppose $\mathbf{X}$ is a hyperbolic Riemann surface with finite genus. Then $\mathbf{X}$ has the HCM-property if and only if $\mathbf{X}$ is either maximal hyperbolic or uniformly perfect.

Proof. The sufficiency is clear from Theorem 1 and the definition of maximal hyperbolic. Now we turn to the necessity. Suppose that $\mathbf{X}$ is not maximal. Again we use Maskit's result [M] to embed $\mathbf{X}$ in a compact Riemann surface $\mathbf{Z}$ with the same genus. If $\mathbf{X}$ is not uniformly perfect, then there is a sequence of points $\left\{a_{n}\right\}$ on $\mathbf{X}$ such that $r_{\mathbf{X}}\left(a_{n}\right) \rightarrow 0$ as $n \rightarrow \infty$. We may assume that $\left\{a_{n}\right\}$ converges to a point $a \in \mathbf{Z}$ since $\mathbf{Z}$ is compact. For sufficiently small $\varepsilon>0$, the surface $\mathbf{Y}=\mathbf{X} \cup\left\{z \in \mathbf{Z}: d_{\mathbf{Z}}(z, a)<\varepsilon\right\}$ is hyperbolic since $\mathbf{X}$ is not maximal (see the proof of Theorem 5). Thus

$$
r_{\mathbf{Y}}\left(a_{n}\right) \geq \delta=r_{\mathbf{Y}}(a) / 2>0
$$


if $n$ is sufficiently large.

Let $K=K(\mathbf{X})(<\infty), f$ be the identity mapping (from $\mathbf{X}$ into $\mathbf{Y}), g$ and $h$ be covering projections of $\mathbf{D}$ onto $\mathbf{X}$ and $\mathbf{Y}$, respectively, and $F: \mathbf{D} \rightarrow \mathbf{D}$ be a lifting of $f$ via $g$ and $h$ so that $f \circ g=h \circ F$. As in the proof of Theorem 2, $\|F\|_{H} \leq K$, so that $F$ is uniformly locally univalent by Lemma 2 , and there is a positive constant $r \leq \delta$ such that $F$ is univalent in each hyperbolic disk of radius $r$.

Select $z_{n} \in \mathbf{D}$ satisfying $g\left(z_{n}\right)=a_{n}$ and let $w_{n}=F\left(z_{n}\right)$, then $h\left(w_{n}\right)=a_{n}$. The Schwarz-Pick Lemma shows that $F$ maps the hyperbolic disk centered at $z_{n}$ with radius $r$ into the hyperbolic disk centered at $w_{n}$ with radius $r$. The radius of the largest hyperbolic disk centered at $z_{n}$ in which $f \circ g$ is univalent is $r_{\mathbf{X}}\left(a_{n}\right)$ $(\rightarrow 0)$. On the other hand, for $n$ sufficiently large, $h \circ F(=f \circ g)$ is univalent in the hyperbolic disk centered at $z_{n}$ with radius $r$, a contradiction.

We now obtain a geometric characterization of the HCM-property which is independent of the genus of the surface. We say that a closed $C^{2}$ curve $\gamma$ on $\mathbf{X}$ belongs to $\Gamma^{*}(\mathbf{X})$ provided $\gamma$ is homotopically nontrivial on $\mathbf{X}$ but there exists another hyperbolic Riemann surface $\mathbf{Y}$ such that $\mathbf{X}$ can be embedded into $\mathbf{Y}$ and $\gamma$ is homotopically trivial on $\mathrm{Y}$ (or more precisely, the image of $\gamma$ under the embedding is homotopically trivial). Roughly speaking, $\Gamma^{*}(\mathbf{X})$ consists of those paths in $\Gamma(\mathbf{X})$ which are homotopically trivial on some larger hyperbolic surface. Define

$$
\mathcal{M}^{*}(\mathbf{X})=\inf \left\{\text { length }_{\mathbf{X}}(\gamma): \gamma \in \Gamma^{*}(\mathbf{X})\right\}
$$

if $\Gamma^{*}(\mathbf{X})$ is nonempty, and

$$
\mathcal{M}^{*}(\mathrm{X})=\infty
$$

if $\Gamma^{*}(\mathbf{X})$ is empty. Clearly, $\mathcal{M}(\mathbf{X}) \leq \mathcal{M}^{*}(\mathbf{X})$ since $\Gamma(\mathbf{X}) \supset \Gamma^{*}(\mathbf{X})$.

Observe that $\Gamma^{*}(\mathbf{X})$ corresponds to the kernel of the induced group homomorphism of the Fuchsian groups uniformizing the surfaces. From this viewpoint, the following Theorem 7 asserts that $\mathrm{X}$ has the HCM-property if and only if there is a positive constant $c$ such that the trace of any element in this kernel is at least $c$.

Theorem 7. If $\mathbf{X}$ is a hyperbolic Riemann surface, then $\mathbf{X}$ has the HCMproperty if and only if $\mathcal{M}^{*}(\mathbf{X})>0$. More precisely, when $\mathcal{M}^{*}(\mathbf{X})$ is finite, the following inequalities hold:

$$
\frac{1}{\tanh \left(\mathcal{M}^{*}(\mathbf{X}) / 4\right)} \leq K(\mathbf{X}) \leq \frac{2}{\tanh \left(\mathcal{M}^{*}(\mathbf{X}) / 4\right)}
$$

Proof. It is easy to show that $\mathbf{X}$ has the HCM-property when $\mathcal{M}^{*}(\mathbf{X})=\infty$. So without loss of generality we may assume that $\mathcal{M}^{*}(\mathbf{X})<\infty$. Hence $\Gamma^{*}(\mathbf{X})$ is nonempty. 
Set $\varepsilon=\mathcal{M}^{*}(\mathbf{X})$. First, we show that $1 / \tanh (\varepsilon / 4) \leq K(\mathbf{X})$. We may assume $K(\mathbf{X})<\infty$. Let $\gamma$ be a closed curve in $\Gamma^{*}(\mathbf{X})$; we only need to show that the hyperbolic length of $\gamma$ is no smaller than $c=4 \operatorname{artanh}(1 / K(\mathbf{X}))$. By definition, $\mathbf{X}$ can be embedded in a hyperbolic Riemann surface $\mathbf{Y}$ such that the image of $\gamma$ is homotopically trivial on $\mathbf{Y}$. Let $f: \mathbf{X} \rightarrow \mathbf{Y}$ be the embedding, $F: \mathbf{D} \rightarrow \mathbf{D}$ be a lifting of $f$ via $g$ and $h$, where $g$ and $h$ denote covering projections of $\mathbf{D}$ onto $\mathbf{X}$ and $\mathbf{Y}$, respectively. As in the proof of Theorem 2, we obtain $\|F\|_{H} \leq K(\mathbf{X})$. Now, Lemma 2 gives $s(F) \geq c / 2$. Let $\delta$ be the lift of $\gamma$ via $g$ with initial point $z$ and terminal point $z^{\prime}$. Then $z \neq z^{\prime}$ since $\gamma$ is homotopically nontrivial on $\mathbf{X}$. On the other hand, $F(z)=F\left(z^{\prime}\right)$ since $F \circ \delta$ is a lift of $f \circ \gamma$ via $h$ and $f \circ \gamma$ is homotopically trivial on $\mathbf{Y}$. Therefore,

$$
\text { length }_{\mathbf{X}}(\gamma)=\text { length }_{\mathbf{D}}(\delta) \geq 2 \mathrm{~s}(\mathrm{~F}) \geq \mathrm{c} .
$$

Next, we show that $K(\mathbf{X}) \leq 2 / \tanh (\varepsilon / 4)$. Let $f$ be an embedding of $\mathbf{X}$ in another hyperbolic Riemann surface $\mathbf{Y}$, and let $F, g$ and $h$ be as above. From Lemma 3(i), it suffices to show that $\|F\|_{H} \leq 2 / \tanh (\varepsilon / 4)$. By Lemma 2, we need only show that $s(F) \geq \varepsilon / 2$. Without loss of generality, we may assume $\varepsilon>0$. If $s(F)<\varepsilon / 2$, then there are two distinct points $z, z^{\prime}$ in $\mathbf{D}$ such that $d_{\mathbf{D}}\left(z, z^{\prime}\right)<\varepsilon$ and $F(z)=F\left(z^{\prime}\right)$. Let $\delta$ be the hyperbolic geodesic joining $z$ and $z^{\prime}$ and $\gamma=g \circ \delta$. Then $f \circ \gamma$ is a homotopically trivial closed curve on $\mathbf{Y}$ since its lifting $F \circ \delta$ via $h$ is a closed curve. On the other hand, $\gamma$ is closed since $f \circ \gamma$ is closed and $f$ is a univalent mapping. Further, $\gamma$ is homotopically nontrivial on $\mathbf{X}$ since its lifting $\delta$ is not closed. Thus, $\gamma$ belongs to $\Gamma^{*}(\mathbf{X})$. But

$$
\text { length }_{\mathbf{X}}(\gamma)=\text { length }_{\mathbf{D}}(\delta)<\varepsilon \text {, }
$$

a contradiction.

One may ask whether the conclusion of Theorem 6 holds for Riemann surfaces of infinite genus, that is, does $\mathbf{X}$ having the HCM-property imply that $\mathbf{X}$ is either maximal or uniformly perfect? The answer is negative as the following example shows.

Example. Let $\mathbf{C}$ be the complex plane. Suppose $G$ and $G^{\prime}$ are the subsets of $\mathbf{C}$ given by

$$
\begin{aligned}
G & =\{z \in \mathbf{C}: 1 \leq \operatorname{Im}(z) \leq 3,|z-(n+2 i)| \geq 1 /|n|, \text { for } n=2,-2,3,-3, \ldots,\}, \\
G^{\prime} & =\{z \in \mathbf{C}:-3 \leq \operatorname{Im}(z) \leq-1,|z-(n-2 i)| \geq 1 /|n|, \text { for } n=2,-2,3,-3, \ldots,\} .
\end{aligned}
$$

The set $G^{\prime}$ is the reflection of $G$ about the real axis. Now we glue $G$ and $G^{\prime}$ together by means of reflection about the real axis to make a Riemann surface $\mathbf{Y}$. Precisely,

1. Identify the points $x+3 i$ with $x-3 i$ for all $x \in(-\infty,+\infty)$. 
2. Identify the points $x+i$ with $x-i$ for all $x \in(-\infty,+\infty)$.

3. Identify the points $(n+2 i)+(1 /|n|) \exp \{i t\}$ with $(n-2 i)+(1 /|n|) \exp \{-i t\}$, where $t \in(-\pi,+\pi], n=2,-2,3,-3, \ldots$.

In other words, the Riemann surface $\mathbf{Y}$ is obtained by taking two copies of $G$ and gluing them together along all boundaries. This produces a hyperelliptic surface of infinite genus.

Then $\mathbf{Y}$ is a hyperbolic Riemann surface with infinite genus. We take $\mathbf{X}$ to be the hyperbolic Riemann surface formed by removing from $\mathbf{Y}$ the closed hyperbolic disk $E=\left\{w \in \mathbf{Y}: d_{\mathbf{Y}}(2 i, w) \leq c\right\}$, where $c$ is any constant satisfying $0<c<r_{\mathbf{Y}}(2 i)$. Clearly, $\mathbf{X}$ is a hyperbolic Riemann surface with infinite genus. We claim that $\mathbf{X}$ has the following properties:

(i) $\mathbf{X}$ is not maximal.

(ii) $\mathbf{X}$ is not uniformly perfect.

(iii) $\mathbf{X}$ has the HCM-property.

We establish these properties in order.

(i) Since $\mathbf{X}$ is properly embedded in $\mathbf{Y}, \mathbf{X}$ is not maximal.

(ii) Let $\mathbf{X}_{n}$ be the annulus $\{z: 1 / n<|z-(n+2 i)|<1\}, n=2,3, \ldots$. It is elementary to calculate that $r\left(\mathbf{X}_{n}\right)=\pi^{2} /(\log n)$. Then for sufficiently large $n$, $\mathbf{X}_{n}$ can be considered as a Riemann surface embedded in $\mathbf{X}$. It is easy to see that $\Gamma(\mathbf{X}) \supset \Gamma\left(\mathbf{X}_{n}\right)$. The principle of hyperbolic metric gives

$$
\mathcal{M}(\mathbf{X}) \leq \mathcal{M}\left(\mathbf{X}_{n}\right)=2 r\left(\mathbf{X}_{n}\right)=2 \pi^{2} /(\log n) \rightarrow 0
$$

as $n \rightarrow \infty$. Thus, $\mathcal{M}(\mathbf{X})=0$ and so $\mathbf{X}$ is not uniformly perfect by Theorem 3 .

(iii) To show that $\mathbf{X}$ has the HCM-property, we need only verify that $\mathcal{M}^{*}(\mathbf{X})>0$. From the construction of $\mathrm{Y}$, it is evident that any closed $C^{2}$ curve on $\mathbf{X}$ which is homotopically nontrivial on $\mathbf{Y}$ does not belong to $\Gamma^{*}(\mathbf{X})$. So all curves in $\Gamma^{*}(\mathbf{X})$ are homotopically trivial on $\mathbf{Y}$. Let $h: \mathbf{D} \rightarrow \mathbf{Y}$ be a covering projection, $\gamma \in \Gamma^{*}(\mathbf{X})$, and $\delta$ be a lifting of $\gamma$ via $h$. Then $\delta$ is closed since $\gamma$ is homotopically trivial on $\mathbf{Y}$. Note that the inverse image of $E$ under $h$ is a union of infinitely many hyperbolic disks on $\mathbf{D}$, each of hyperbolic radius $c$. Also, $\delta$ is homotopically nontrivial on $\mathbf{D} \backslash h^{-1}(E)$ since $\gamma=h \circ \delta$ is homotopically nontrivial on $\mathbf{X}$. Then $\delta$ must surround at least one of the hyperbolic disks in $h^{-1}(E)$. Consequently, by the principle of hyperbolic metric,

$$
\text { length }_{\mathbf{X}}(\gamma) \geq \text { length }_{\mathbf{Y}}(\gamma)=\text { length }_{\mathbf{D}}(\delta) \geq \mathrm{c} .
$$

Therefore, $\mathcal{M}^{*}(\mathbf{X}) \geq c>0$, or $\mathbf{X}$ has the HCM-property.

Remark. Note that every maximal hyperbolic Riemann surface with finite genus $\mathbf{g} \geq 2$ is uniformly perfect. The preceding example shows that this does not remain valid for infinite genus. It is not difficult to show that the Riemann surface $\mathbf{Y}$ constructed above gives us an example of a maximal hyperbolic Riemann surface with infinite genus that is not uniformly perfect. 


\section{References}

[BP] Beardon, A.F., and Ch. Pommerenke: The Poincaré metric of plane domains. - J. London Math. Soc. (2) 18, 1978, 475-483.

[B] Bochner, S.: Fortsetzung Riemannscher Flächen. - Math. Ann. 98, 1927, 406-421.

[H] HaIfawa, M.M.: On the behaviour of hyperbolic curvature under univalent bounded transformation. - Rend. Circ. Mat. Palermo (2) 16, 1967, 57-63.

[FO] FlinN, B., and B. Osgood: Hyperbolic curvature and conformal mapping. - Bull. London Math. Soc. 18, 1986, 272-276.

[L] LiU, X.: Topics in geometric function theory. - Ph.D. dissertation, University of Cincinnati, Cincinnati, Ohio (in preparation).

$\left[\mathrm{MM}_{1}\right] \mathrm{MA}, \mathrm{W}$. , and D. Minda: Euclidean linear invariance and uniform local convexity. - J. Australian Math. Soc. (to appear).

$\left[\mathrm{MM}_{2}\right] \mathrm{MA}, \mathrm{W}$., and D. Minda: Hyperbolic linear invariance and hyperbolic $k$-convexity. - To appear.

[MRR] MaRden, A., I. Richards, and B. Rodin: Analytic self-mappings of Riemann surfaces. - J. Analyse Math. 18, 1967, 197-225.

[M] Maskit, B.: The conformal group of a plane domain. - Amer. J. Math. 90, 1968, 718-722.

[Mi] Minda, D.: Hyperbolic curvature on Riemann surfaces. - Complex Variables Theory Appl. $12,1989,1-8$.

[O] Oнtsuka, M.: On the behavior of an analytic function about an isolated boundary point. - Nagoya Math. J. 4, 1952, 103-108.

[P] PICK, G.: Über die konforme Abbildung eines Kreises auf ein schlichtes und zugleich beschränktes Gebiet. - S.-B. Kaiserl. Akad. Wiss. Wien. 126, 1917, 247-263.

[Po $\left.{ }_{1}\right]$ Pommerenke, Ch.: Linear-invariante Familien analytischer Funktionen I. - Math. Ann. $155,1964,108-154$.

[Po $\left.{ }_{2}\right]$ Pommerenke, Ch.: Uniformly perfect sets and the Poincaré metric. - Arch. Math. 32, 1979, 192-199.

Xiangyang Liu

University of Cincinnati

Department of Mathematical Sciences

Cincinnati, Ohio 45221-0025

U.S.A.

Received 20 July 1990
David Minda

University of Cincinnati

Department of Mathematical Sciences

Cincinnati, Ohio 45221-0025

U.S.A. 